\title{
Exploration on Signal Processing Performance of Wireless Sensor in Electromagnetic Interference Environment
}

\author{
Zhuorui Yi $\mathbb{D}^{D}$, Tao Gu $\mathbb{D}^{\text {, }}$, and Qing Ma \\ Chongqing University, Chongqing City, 400044, China \\ Correspondence should be addressed to Zhuorui Yi; 20192480@cqu.edu.cn
}

Received 4 January 2022; Revised 26 January 2022; Accepted 15 February 2022; Published 7 March 2022

Academic Editor: Haibin Lv

Copyright (C) 2022 Zhuorui Yi et al. This is an open access article distributed under the Creative Commons Attribution License, which permits unrestricted use, distribution, and reproduction in any medium, provided the original work is properly cited.

\begin{abstract}
In order to deeply analyze the performance of Electromagnetic Field (EMF) on wireless sensor Signal Processing (SP), this paper first examines the influencing factors of wireless sensor SP performance based on the relevant theories of EMF and wireless sensor. Then, it discusses the influence of EMF on wireless sensor information Transmission and Reception (T \& R) time, Package Reception Rate (PRR), and communication throughput. Furthermore, the Packet Loss Rate (PLR) and Bit Error Rate (BER) of the sensor are described, and finally, a conclusion is drawn. The research findings are as follows. This paper makes a statistical study on whether the wireless sensor is disturbed by EMI from three aspects (wireless sensor's information T \& R time, PLR, and communication throughput). In any case, EMF will prolong the wireless sensor's information $\mathrm{T} \& \mathrm{R}$ time and reduce the PRR and communication throughput. When the distance between the transmitter and the receiver is set to $5 \mathrm{~m}$, the change of field intensity at the transmitter significantly impacts the BER but has little impact on the PLR. However, when the field intensity of the transmitter and the receiver is the same, PLR and BER show no evident difference. Therefore, this paper studies the SP performance of the wireless sensor in the EMI environment, which provides an excellent reference significance for the rapid development of wireless sensors in the future.
\end{abstract}

\section{Introduction}

With Science and Technology (S \& T) advancement, Wireless Sensor Technology (WST) is seeing a more important role in production and life. At the same time, the wireless sensors are always interfered with by the Electromagnetic (EM) Field (EMF) [1]. Therefore, how to reduce the impact of EMF on wireless sensors' Signal Processing (SP) performance has become one of the major problems [2,3]. Mehran et al. optimized the concept of EM Compatibility (EMC) and proposed three necessary conditions for EM compatible systems: (1) The system would not affect or interfere with other systems. (2) The system would not be affected by other systems' radiation. (3) The system would not interfere with breeding. EMC can solve EM Interference (EMI) in wireless sensor signals [4]. Yu et al. optimized the wireless sensororiented SP method using the additive equivalence principle. They concluded that the farther the EMF was, the smaller its impact on the wireless sensors' SP performance. Additionally, the researchers calculated the EMF wires' specifications.
They found that $8 \times$ LGJ-300/40 wires had relatively little impact on the wireless sensor signal, providing a sure basis for selecting the EMF wire model [5]. Abdel et al. proposed to analyze the EMF influence on wireless sensors' SP performance by a boundary element method. The experiment had chosen a single-circuit transmission line, suitable for traditional power grids and inconsistent with real-life applications. Consequently, the proposed scheme was not applied in practice [6]. Li et al. optimized the strategy of Abdel et al. and introduced multicircuit and multisplit transmission lines instead of the single-circuit transmission line and discovered that the ground EMF Intensity (INT) would increase with the increase of the number of split transmission lines and the distance between the EMF [7].

In this context, the present work starts with the wireless sensors' SP performance expounds on the influence of EMF on wireless sensors from information Transmission and Reception time, information reception rate, and communication throughput. Then, it deeply analyzes wireless sensors' Bit Error Rate (BER) and Packet Loss Rate (PLR) variation 
in different fields of view. The above experts and scholars have studied the SP performance of WST in the EMI environment from different angles. Still, they have not analyzed it from all aspects, which has a certain-sidedness. According to a particular theoretical logical order, the present work comprehensively analyzes the research on wireless sensor SP performance in an EMI environment using comparative analysis and other methods. It attempts to provide a methodological reference for the research on wireless sensor SP performance in the EMI environment. Figure 1 unfolds the specific structure of this paper.

\section{EMF and Wireless Sensor Theory}

2.1. Basic Principle of EMF. EMW is a movement form of EMF, but in high-frequency electric oscillation, magnetoelectric interaction is swift, and all energy cannot return to the original oscillation circuit. As a result, Electric Energy (EEIX) and magnetic energy propagate to space through EMW with the periodic transformation of $\mathrm{EF}$ and $\mathrm{MF}$ [8-10]. The EMW is a shear wave, and the MF, EF, and traveling direction of the EMW are perpendicular to one another [11-13]. The propagation of EMW includes ground waves propagating and airwaves propagating. The longer the Wavelength (WL) of the ground wave is, the less its attenuation is [14-16]. The longer the WL is, the easier it is to bypass obstacles and propagate farther $[17,18]$.

MWE is one of the core methods to study EMF, mainly composed of four theorems: Ampere's Circuital Law (ACL), EM induction law, Gauss's law of electric flux, and Gauss's law of magnetic flux [19].

2.1.1. ACL.

$$
\oint B \cdot d l=\mu_{0} I
$$

Equation (1) represents the loop integral of the magnetic field INT in the magnetic field along any closed directed curve, equal to the sum of the currents passing through the curved surface determined by the closed integral path. $\mu_{0}$ denotes the permeability in a vacuum. $I$ indicates the wire current. $d l$ stands for the line element on $l$. B is the magnetic field.

\subsubsection{EM Induction Law.}

$$
E=-\frac{n(\Delta \phi)}{(\Delta t)}
$$

The EM induction law is also known as Faraday's law of EM induction. $n$ represents the number of coil turns. $(\Delta \phi) /$ $(\Delta t)$ indicates induced electromotive Potential Energy (PE). In physics, induced electromotive PE can be expressed by letters $\varepsilon$, as calculated in

$$
\varepsilon=\frac{\Delta \phi}{\Delta t}
$$

In Equation (3), $\Delta t$ represents the time cost for the change, and $\Delta \phi$ indicates the magnetic flux change.

2.1.3. Gauss's Law of Magnetic Flux. Gauss flux law describes that the magnetic flux generated through any closed surface in an MF equals zero. Its differential form is calculated by

$$
\nabla \cdot B=0
$$

In Equation (4), $B$ represents the MF. The integral form is calculated by Equation (5).

$$
\oint_{s} B \cdot d a=0
$$

In Equation (5), $s$ represents a closed surface. $d a$ denotes a small area fraction. The remaining letters share the same meaning as those in the above equations. Gauss's law of magnetic flux states that the vector line of magnetic induction INT is a continuous and uninterrupted closed vector line, showing that the magnetic field is passive.

2.1.4. Gauss's Law of Electric Flux. Gauss's law of electric flux describes that the electric flux generated through any closed surface in an EM equals the amount of charge surrounded by the surface [20]. Its differential form is calculated by

$$
\oint_{s} D \cdot d a=q .
$$

In Equation (6), $D$ and $q$ represent the electric flux and the amount of charge, respectively, and the remaining letters share the same meaning as those in the above equations.

2.2. EMF Differential Equations. The above equations need further optimization to be used in actual production and life. Equation (7) can be obtained by introducing the displacement current assumption.

$$
\left\{\begin{array}{l}
\nabla \times R=L+\frac{\partial \phi}{\partial t}, \\
\nabla \times F=\frac{\partial B}{\partial t}, \\
\nabla \cdot T=\rho, \\
\nabla \cdot B=0 .
\end{array}\right.
$$

Equation (7) calculates the differential forms of ampere loop law, EM induction law, Gauss's law of magnetic flux, and Gauss's law of electric flux, respectively. $R, L, F, T$, and $\rho$ represent magnetic field INT, current density, electric field INT, potential shift vector, and the charge density, respectively. $\partial \phi / \partial t$ signifies the partial derivative of induced electromotive Potential Energy, and the remaining letters share the same meaning as those in the above equations. 


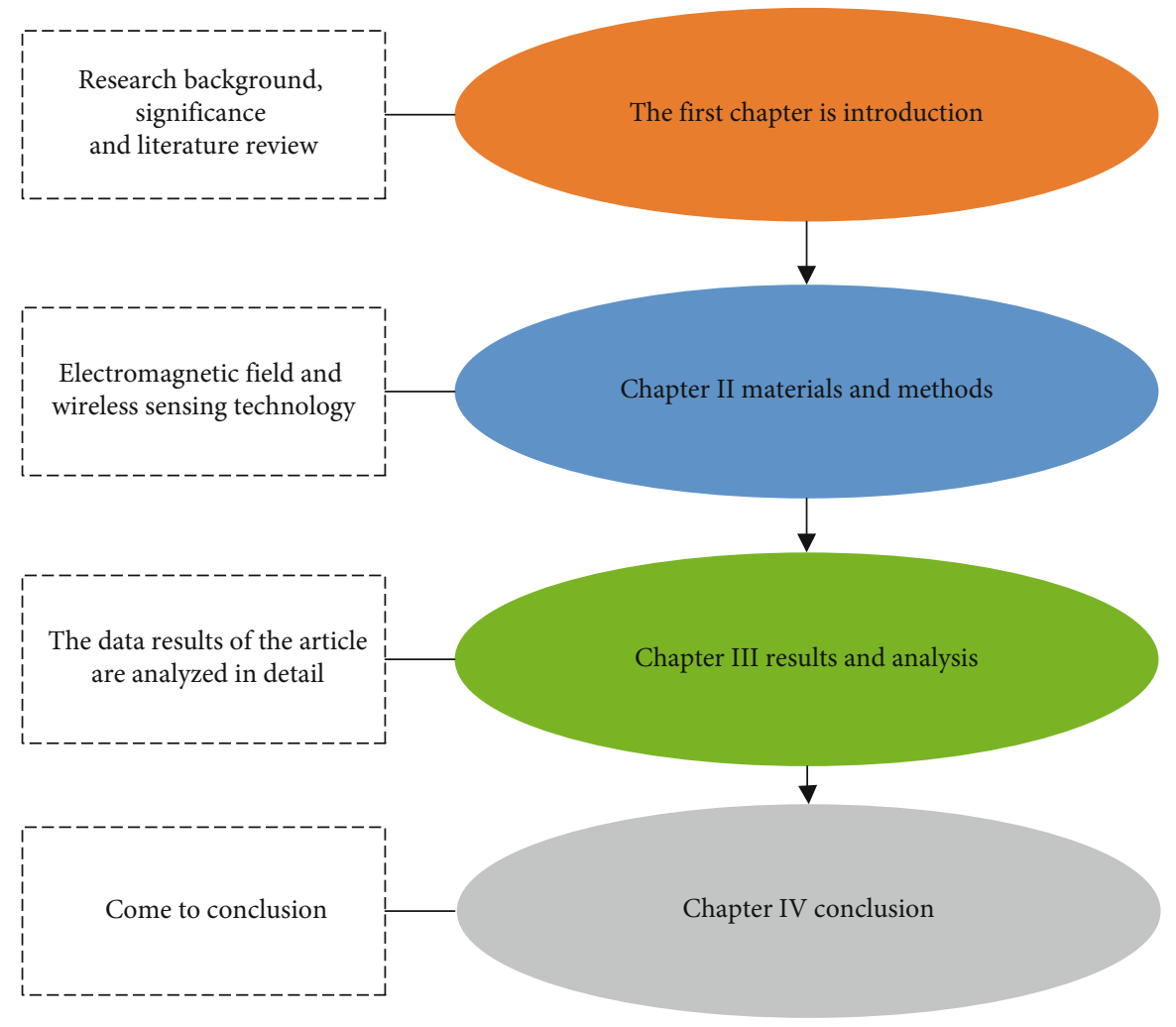

FIgURE 1: Paper organization structure.

Further, Equation (7) can be simplified as

$$
\left\{\begin{array}{l}
T=\varepsilon F, \\
B=\mu R \\
L=\sigma F .
\end{array}\right.
$$

In Equation (8), $\varepsilon, \mu$, and $\sigma$ represent the dielectric constant, magnetic permeability, and conductivity, respectively. The remaining letters share the same meaning as those in the above equations.

The EF and MF can be analyzed separately to simplify the EMF calculation and analysis. Firstly, two different variables need to be introduced: Vector Magnetic Potential (VMP) and Electric Scalar Potential (ESP) [21]. Equation (9) displays the specific calculation form.

VMP is as follows:

$$
B=\nabla \cdot A
$$

In Equation (9), $A$ represents VMP, and the remaining letters share the same meaning as those in the above equations.

ESP is follows:

$$
F=-\nabla \varnothing
$$

In Equation (10), $\varnothing$ represents ESP, and the remaining letters share the same meaning as those in the above equations.
Equations (9) and (10) suggest that the VMP and ESP are related to the four theorems in Section 2.1 and, thus, can be applied to them. The specific forms are shown in Equations (11) and (12).

MF DE is as follows:

$$
\nabla^{2} A-\mu \varepsilon \frac{\partial^{2} A}{\partial t^{2}}=-\mu L
$$

In Equation (11), $\nabla^{2}$ represents Laplacian, and the remaining letters share the same meaning as those in the above equations.

$\mathrm{EF} \mathrm{DE}$ is as follows:

$$
\nabla^{2} \phi-\mu \varepsilon \frac{\partial^{2} \phi}{\partial t^{2}}=-\frac{\rho}{\varepsilon}
$$

In Equation (12), the letters share the same meaning as those in the above equations.

Based on Equations (11) and (12), the expression of MF $\mathrm{DE}$ and EF DE is similar, and the left and right sides of the equations are symmetrical. Therefore, Equation (13) can be obtained through the Lorentz condition.

$$
\nabla \cdot A=-\mu \varepsilon \frac{\partial \varnothing}{\partial t} .
$$

In Equation (13), the letters share the same meaning as those in the above equations. 
The charge in the EMF will change most of the time. Yet it stops changing sometimes and triggers Electrostatic Field (ESF). Accordingly, Equation (14) can be obtained.

$$
\left\{\begin{array}{l}
\nabla \times R=L, \\
\nabla \times F=0, \\
\nabla \cdot B=\rho, \\
\nabla \cdot D=0 .
\end{array}\right.
$$

In Equation (14), the letters share the same meaning as those in the above equations.

Specifically, ESF refers to the EF observed under the relatively fixed charge not changing with time. Then, Equations (15) and (16) can be obtained based on the definition of ESF and mathematical model.

$$
\begin{gathered}
\nabla \times F=0, \\
\nabla \times D=\rho .
\end{gathered}
$$

2.3. Boundary Conditions (BCs) in EMF Interference Environment. The real solution of EMF must be constrained by the given BCs and Initial Conditions (ICs) to get a practical solution. BCs mainly involve (1) Dirichlet BC, (2) Neumann BC, and (3) mixed BC [22].

Equations (17) and (18) calculate the Dirichlet BC.

The calculation of free charge DENS region reads

$$
\nabla^{2} \varphi=-\frac{\rho}{\varepsilon}
$$
reads

The calculation of the DENS region without free charge

$$
\nabla^{2} \varphi=0
$$

In Equations (17) and (18), $\varphi$ represents the Dirichlet $\mathrm{BC}$, and the remaining letters share the same meaning as those in the above equations.

If the surface potential of each conductor is given, Equation (19) can be obtained.

$$
\varphi_{r_{i}}=C_{i}
$$

In Equation (19), $r_{i}$ is the surface potential of any conductor, and $C_{i}$ is a known constant.

Equation (20) calculates the connection conditions when there is no free surface charge on the interface of different media:

$$
D_{2 n}=D_{1 n} \text { or }\left.\varepsilon_{1} \frac{\partial \varphi_{1}}{\partial n}\right|_{n}=\left.\varepsilon_{2} \frac{\partial \varphi_{2}}{\partial n}\right|_{n}
$$

Equation (21) calculates the connection conditions when there is a free surface charge on the interface of different media:

$$
F_{2 r}=F_{1 t} \text { or }\left.\varphi_{1}\right|_{n}=\left.\varphi_{2}\right|_{n}
$$

When the charge is distributed in a finite space, the potential at the infinite boundary of the specified field is zero, as manifested in

$$
\varphi_{\infty}=0
$$

The Neumann BC is calculated by Equations (23) and (24).

The calculation of free charge DENS region reads

$$
\nabla^{2} \varphi=-
$$
reads

The calculation of the DENS region without free charge

$$
\nabla^{2} \varphi=0
$$

If the surface potential of each conductor is given, Equation (25) is obtained:

$$
-\left.\varepsilon \frac{\partial \varphi}{\partial n}\right|_{n}=\sigma_{i}
$$

In Equation (25), $\sigma_{i}$ represents a known function.

If the total charge of each conductor is constant, Equation (26) can be obtained:

$$
\oint-\varepsilon \frac{\partial \varphi}{\partial n} \mathrm{~d} s=q_{i}
$$

In Equation (26), $q_{i}$ is a known value, and the conductor is an allele.

The mixed BC is calculated by Equations (27) and (28).

The calculation of free charge DENS region reads

$$
\nabla^{2} \varphi=-\frac{\rho}{\varepsilon}
$$

The calculation of the DENS region without free charge reads

$$
\nabla^{2} \varphi=0
$$

If the surface potential of each conductor is given, Equation (29) can be obtained:

$$
\varphi_{\mathrm{r}_{i}}=C_{i}
$$

2.4. Wireless Sensor Network (WSN) System Architecture. WSN mainly comprises data acquisition, data promulgation network, and control management center. Its operation principle integrates the sensor nodes with processing units and communication modules. Then, a distributed network composed of nodes is formed through preset protocols. Finally, the collected data are transmitted to the Information 


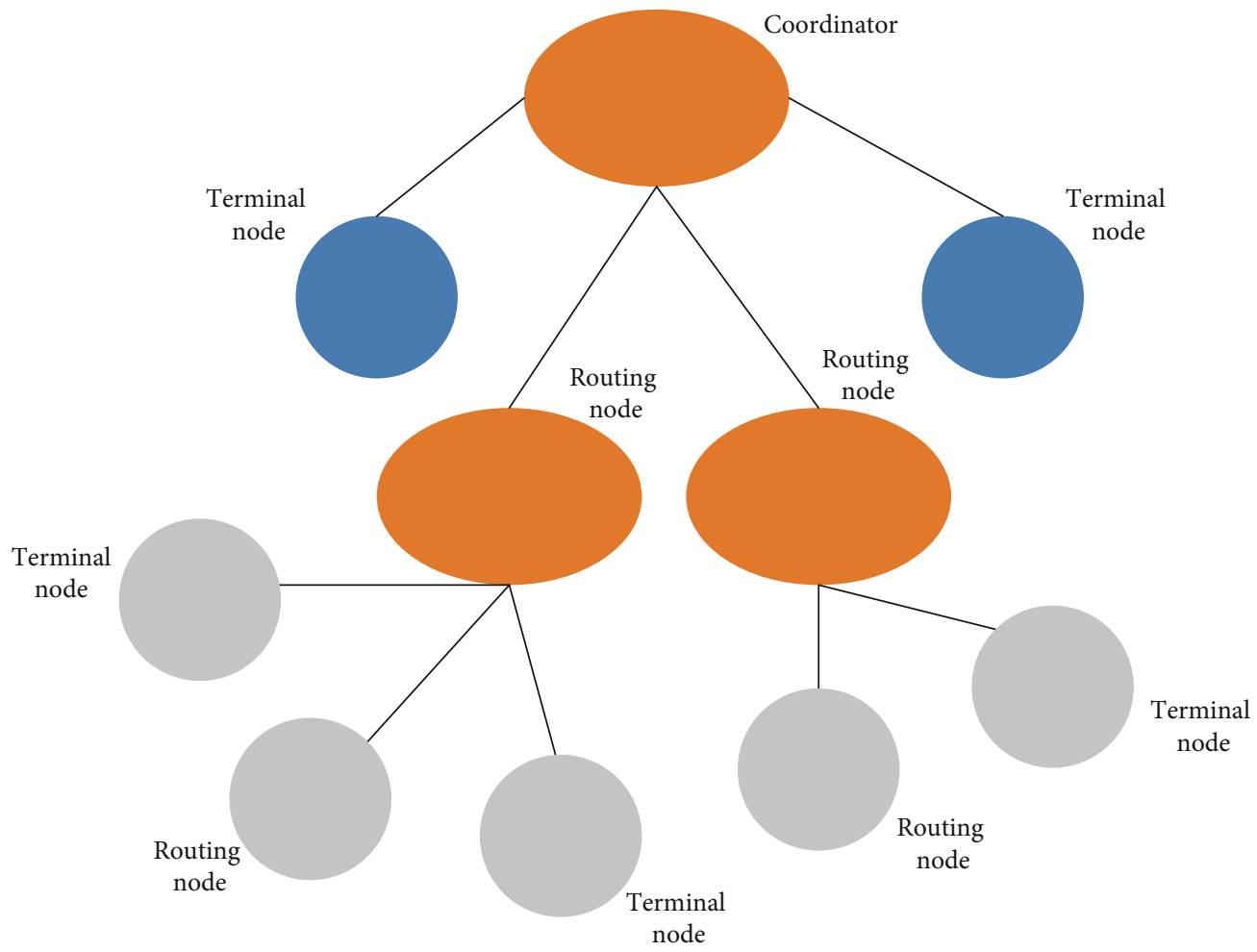

FIGURE 2: WSN system architecture.

Processing Center (IPC) through radio waves after optimization [23]. Figure 2 depicts the specific system structure.

Figure 3 Outline of the characteristics of wireless sensors.

Figure 3 unfolds the wireless sensor features: limited hardware resources, limited power capacity, centerless selforganization, multihop routing, dynamic topology, a large number of nodes, limited transmission capacity, and security problems.

Figure 4 divides the threats to WSN into four categories.

Figure 4 classifies the threats to wireless sensor networks into four types: Type I (congestion attack and physical destruction), Type II (collision attack, depletion attack, and unfair competition), Type II (discarding, greedy destruction, and misguided directional attack, and Type IV (flooding attack and synchronous destruction attack).

2.5. Influencing Factor Analysis of Wireless Sensor. An active EMF will produce EMW and trigger interferences to wireless sensors. Wireless sensors receive one kind of interference through the antenna; the other generates coupling on the line inside the wireless sensor, directly affecting the internal circuits [24].

2.5.1. Analysis of Influence on Wireless Sensor Antenna. Then, this section chooses the dipole antenna for the wireless sensor, as drawn in Figure 5.

Equation (30) displays the specific calculation method of induced voltage INT based on Figure 4 analysis.

$$
Y=H V .
$$

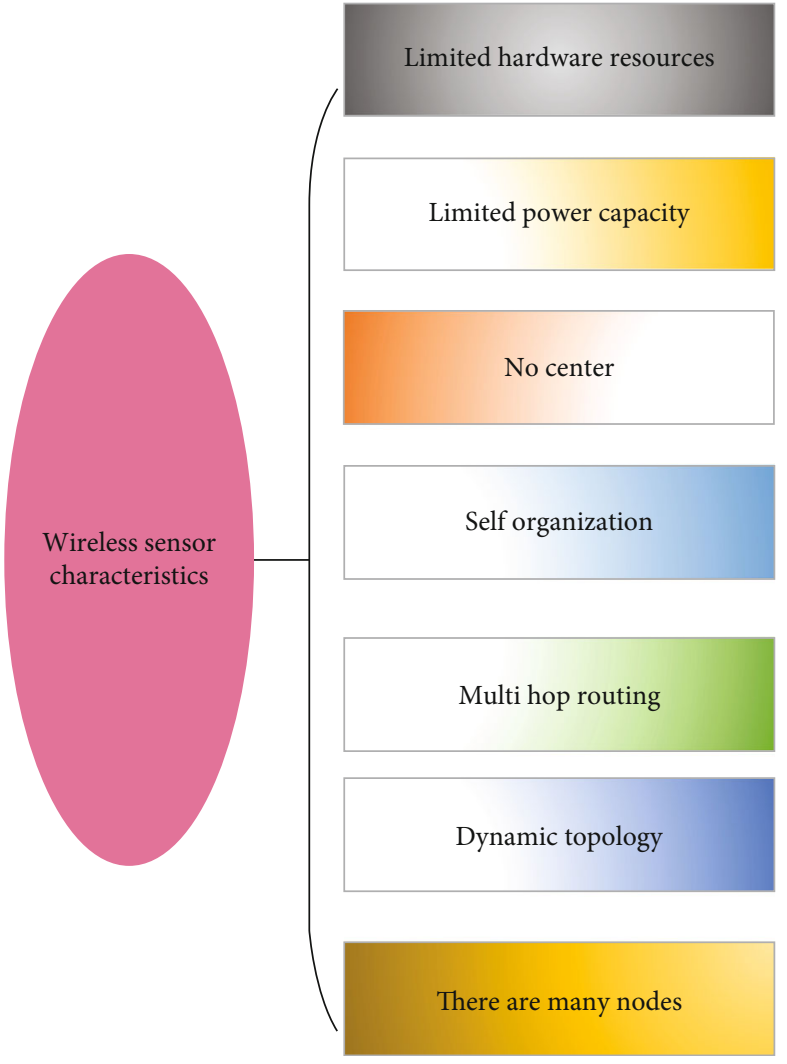

FIgURE 3: Wireless sensor characteristics. 


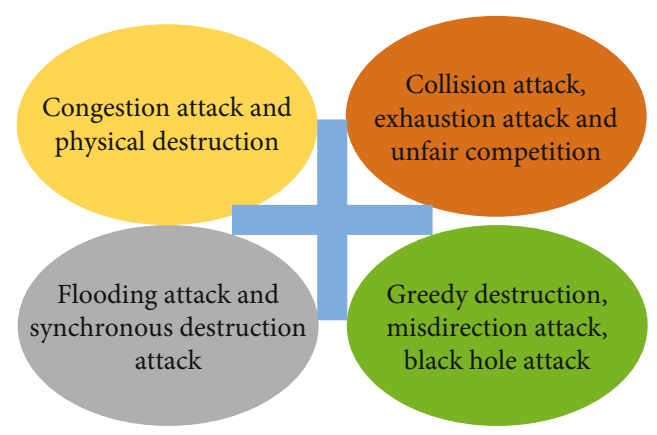

Figure 4: Threats to WSN.

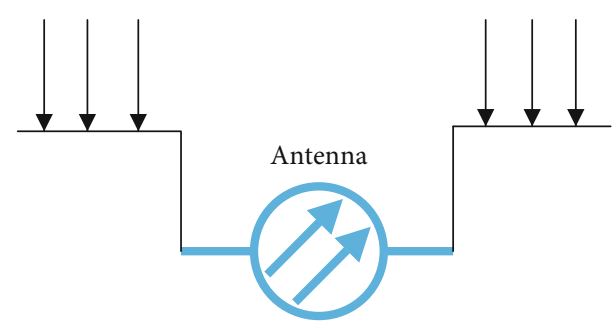

Figure 5: Receiving antenna model.

In Equation (30), $Y, H$, and $V$ represent the induced voltage OMT, the antenna length, and the horizontal INT, respectively.

Figure 6 can be obtained by replacing the antenna model with a typical circuit.

Equation (31) can calculate the interference current.

$$
Q=\frac{Y}{Z_{1}+Z_{2}} .
$$

In Equation (31), $Z_{1}, Z_{2}$, and $Q$ indicate internal load impedance, antenna impedance, and interference current, and the remaining letters share the same meaning as those in the above equations.

2.5.2. Impact Analysis on the Internal Loop of Wireless Sensor. In real life, the antenna effect is jointly affected by the radiation field signal's WL and the sensor's internal circuit size. Figure 7 specifies the coil induction principle.

Figure 7 shows that a current flowing through a wire will generate an EMF around the wire, and the wire itself in the EMF will induce other wires within the EMF. Obviously, a variable EMF in the closed-circuit will generate an induced voltage on the circuit. Equation (32) specifies the calculation method.

$$
Y=-\frac{d}{d t} \int B d s
$$

In Equation (32), $B, s$, and $Y$ represent the magnetic field INT, the loop area, and the induced voltage INT, and the remaining letters share the same meaning as those in the above equations.

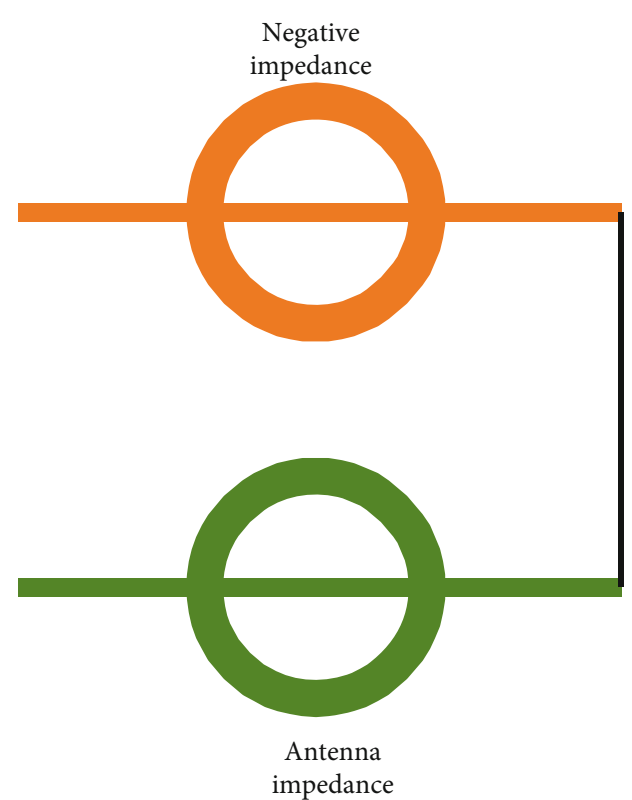

Figure 6: Equivalent circuit diagram of antenna model.

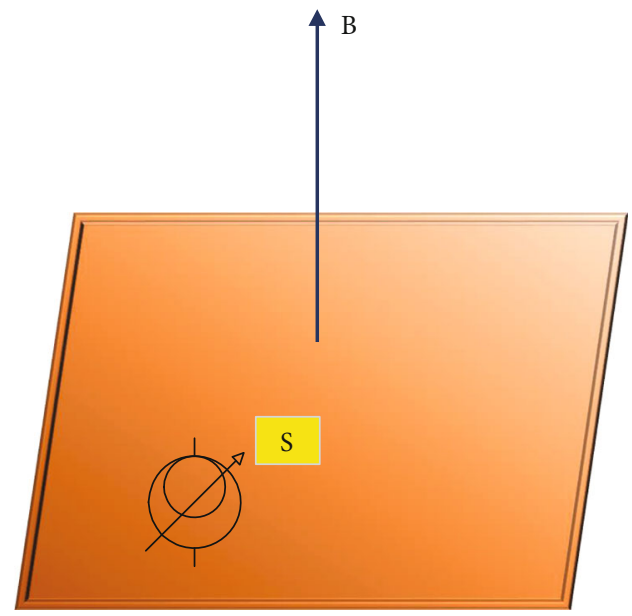

Figure 7: Coil induction principle.

2.6. Adopted Research Methods. The following are the adopted research methods:

(1) Literature review method: it refers to the comprehensive introduction and elaboration of articles through reading, analysis, induction, and sorting out the latest progress, academic opinions, or suggestions of current topics, problems, or research topics [25].

(2) Quantitative and qualitative analysis: it refers to a standard method to analyze, study, and summarize quantity characteristics, the logical relationship between quantities, and the changing trend of quantity by collecting relevant research data. Specifically, qualitative analysis refers to how the forecaster analyzes the future development trend, change, and nature according to relevant data changes over the 


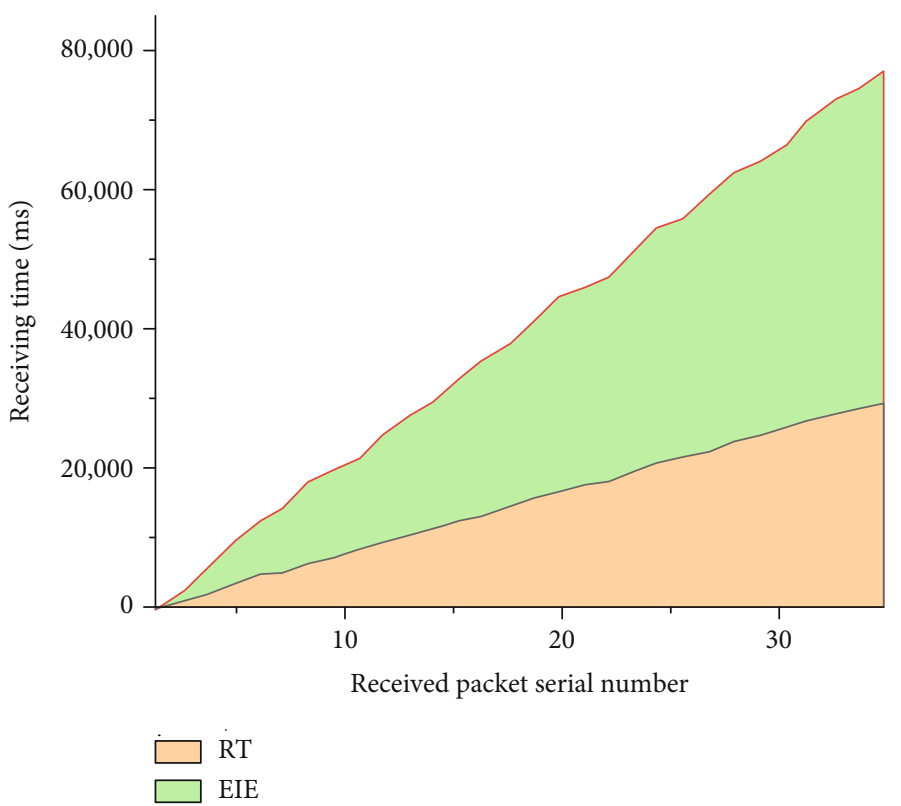

FIGURE 8: Statistics of the wireless sensor's signal receiving time in the same environment (RT indicates that the wireless sensor is free of EMI, and EIE means that the wireless sensor is subject to EMI).

years, government policy releases, and influential major social events [26, 27].

(3) Simulated charge method: the principle is to equivalently replace the spatially continuously distributed charges with a limited number of discrete charges arranged at a specific geometric position. Then, it calculates the potential distribution and EF INT from these discrete charges according to the superposition principle $[28,29]$.

(4) Boundary element method: the boundary is discretized into a series of elements. The boundary value problem is equivalent to the boundary integral equation problem. Then, the finite element discretization technology is used to solve the field problem [30-33].

\section{Analysis of EMF Interference on Wireless Sensor SP Performance}

3.1. Influence of EMF on the Wireless Sensor from Different Perspectives. This section starts from the following points to study and analyze the influence of EMF on wireless sensor's SP performance:

3.1.1. Sending and Receiving Time of Wireless Sensor Signal. Firstly, the computer is used to set the signal sending interval to one second. It counts the time when the other party receives 35 pieces of information, and finally, it measures the results in the environment with or without EMI. Figure 8 shows the specific experimental results.

As illustrated in Figure 8, the signal receiving time of wireless sensors in the environment with or without EMI shows an increasing trend. The maximum signal receiving time without EMI is $30,000 \mathrm{~ms}$, and the received packet
Serial Number (SN) is 35. The maximum signal receiving time under EMI is about $72,000 \mathrm{~ms}$, and the received packet $\mathrm{SN}$ is also 35. This data group suggests that the EMF can prolong the wireless sensor's signal receiving time by about 42,000 ms.

Additionally, Figure 9 shows the influence of EMF on the wireless sensor in different environments.

Figure 9 is similar to Figure 7 in data changes. The wireless sensor's signal receiving interval shows an increasing trend regardless of EMI. In different environments, the wireless sensor's maximum signal receiving time without EMI is $30,000 \mathrm{~ms}$, and the received package $\mathrm{SN}$ is 35 . Meanwhile, the wireless sensor's maximum signal receiving time under EMI is about $96,000 \mathrm{~ms}$. At this time, the received packet $\mathrm{SN}$ is also 35. Apparently, under EMI, the wireless sensor's signal receiving time is extended by about $66,000 \mathrm{~ms}$.

3.1.2. Wireless Sensor's Package Reception Rate (PRR). Firstly, the computer is used to set the signal sending interval of the wireless sensor to $100 \mathrm{~ms}$. It counts the time when the other party receives 35 pieces of information, and finally, it counts the results in the environment with or without EMI. Figure 10 specifies the experimental results.

Figure 10 proves that the PRR of the wireless sensor in the environment with and without EMI is constantly fluctuating. In the same environment, the PRR of the wireless sensor without EMI fluctuates between $72 \%$ and $100 \%$, with 16 times $100 \%$ and once $70 \%$. Under the EMI, the PRR of wireless sensor fluctuates between $35 \%$ and $81 \%$, with one time $81 \%$ and $35 \%$ PRR each.

Figure 11 reflects the influence of EMI on the wireless sensor in different environments.

The data change in Figure 11 is similar to that in Figure 9, constantly fluctuating. The PRR of the wireless sensor without EMI in different environments is basically the 


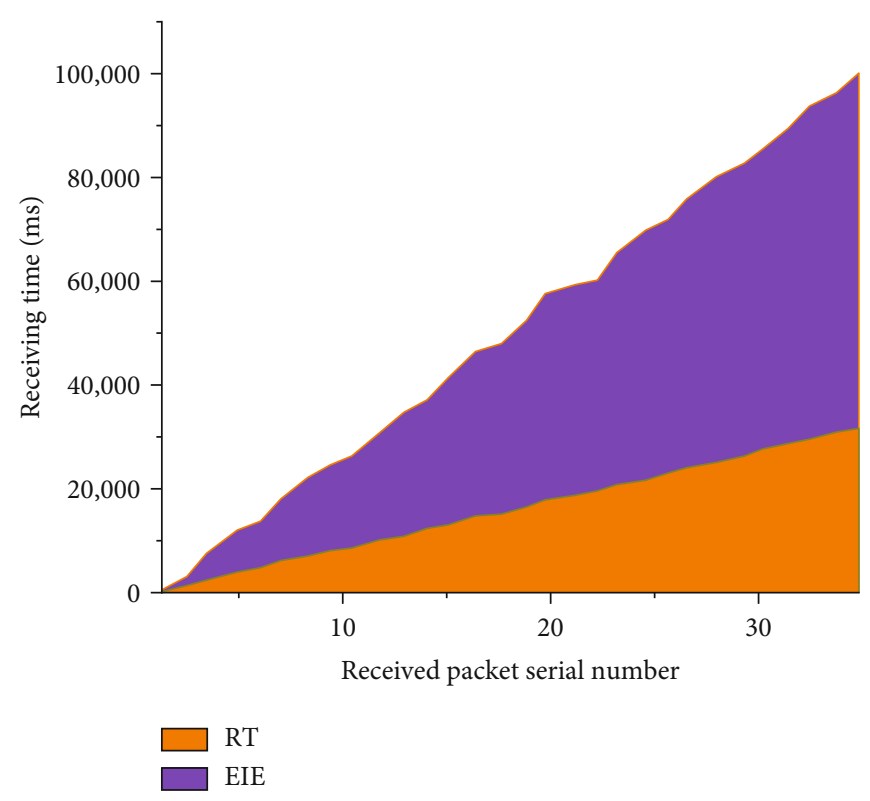

FIGURE 9: Statistics of wireless sensor's signal receiving time in different environments.

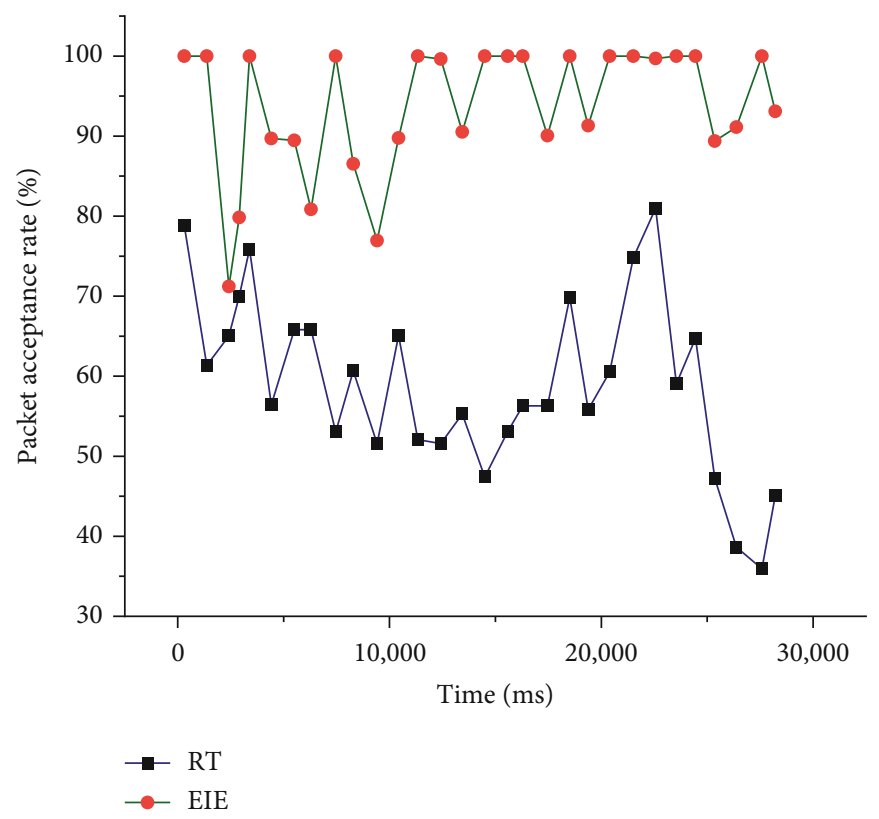

FIGURE 10: Statistics of wireless sensor's PRR in the same environment.

same as that in the same environment. Under EMI, the wireless sensor's PR fluctuates between $10 \%$ and $68 \%$, with one time $68 \%$ PRR and twice $10 \%$ PRR.

\subsubsection{Wireless Sensor's Communication Throughput.} Figure 12 first fixes the signal sending interval of the wireless sensor at $5 \mathrm{~ms}$, counts the information received by the system within one second, and finally calculates the communication throughput in the environment with or without EMI.

As described in Figure 12, the wireless sensor communication throughput in the same environment constantly fluctuates. The communication throughput in the environment without EMI is between 2,400 and 3,100, and the highest point is between $1,500 \mathrm{~ms}$ and $20,000 \mathrm{~ms}$. The communication throughput under the EMI environment is $1,700 \sim 2,300$, the highest point is between $5,000 \mathrm{~ms}$ and $10,000 \mathrm{~ms}$, and the lowest point is between $25,000 \mathrm{~ms}$ and $30,000 \mathrm{~ms}$.

Figure 13 details the influence of EMI on wireless sensor communication throughput in different environments.

The wireless sensor's communication throughput without EMI in different environments is similar to Figure 12. The wireless sensor's communication throughput with EMI is between 1,400 and 2,100, the lowest point is between 


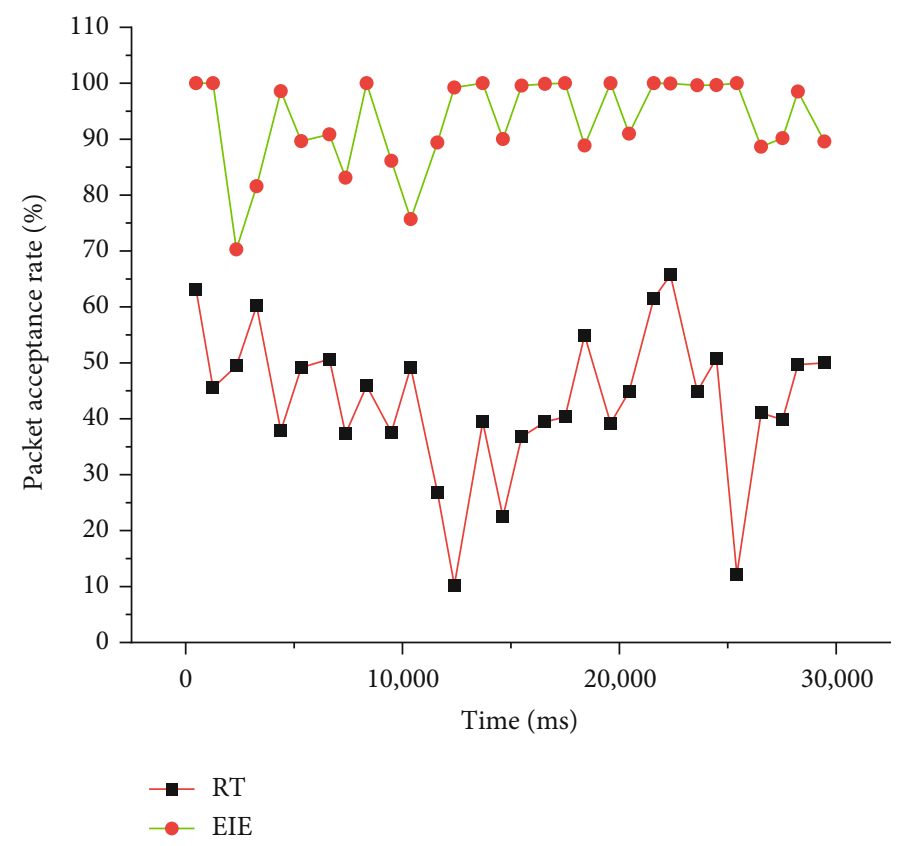

FIGURE 11: Statistics of wireless sensor's PRR in different environments.

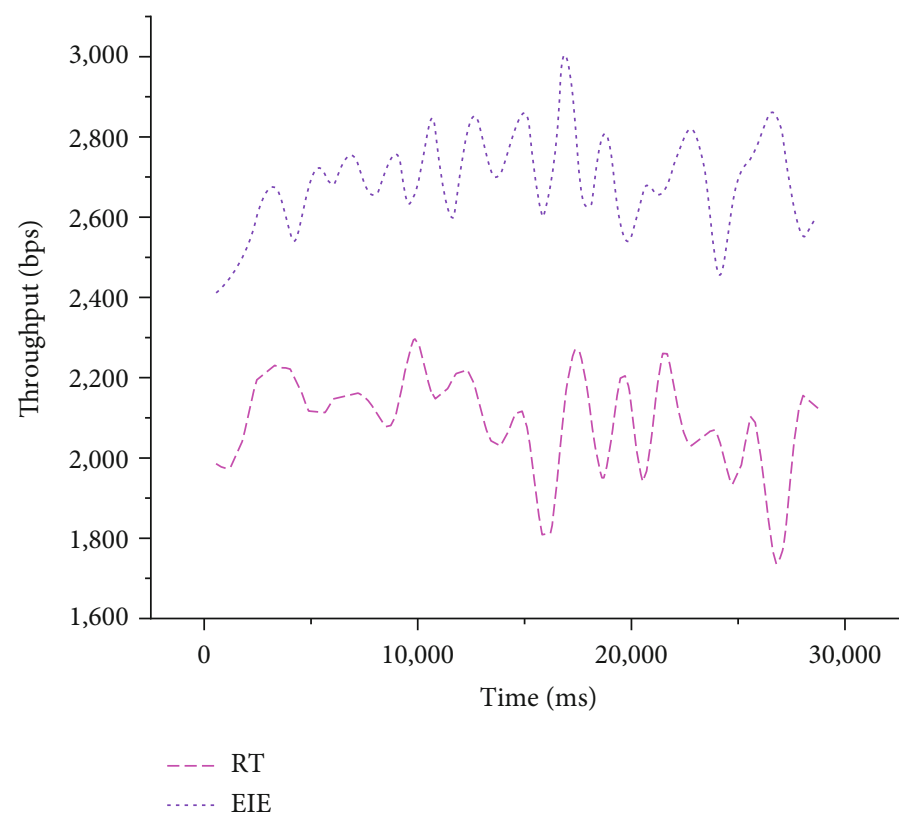

FIGURE 12: Statistics of wireless sensor's communication throughput results in the same environment.

$15,000 \mathrm{~ms}$ and $20,000 \mathrm{~ms}$, and the highest point is near $18,000 \mathrm{~ms}$ and $21,000 \mathrm{~ms}$.

To sum up, the experiment statistically studies whether the wireless sensor is disturbed by EMI from three aspects: wireless sensor's Transmission and Reception ( $\mathrm{T} \& \mathrm{R})$ time, PRR, and communication throughput. The findings show that EMI will more or less prolong the wireless sensor's signal receiving time under any circumstances. The PRR and communication throughput are reduced.

3.2. SP Performance of the Wireless Sensor. The above experiments study the wireless sensor's T \& R time, PRR, and communication throughput information. Next, it is necessary to check wireless sensors' SP performance, as drawn in Figure 14.

Figure 14 implies that when the transmitting and receiving EMF INT are consistent, there is a linear relationship between the EMF span [denoted by the distance between the signal Transmitter and Receiver of the wireless sensor] and the wireless sensor's PLR and BER. With the increase of the ENF span, the PLR and BER show a rising trend. When the EMF span is $10 \mathrm{~m}$, the BER is about $6.55 \%$, increasing by about $1 \%$. At this time, the PLR is about $1.72 \%$, increased by about $0.8 \%$. Hence, the difference 


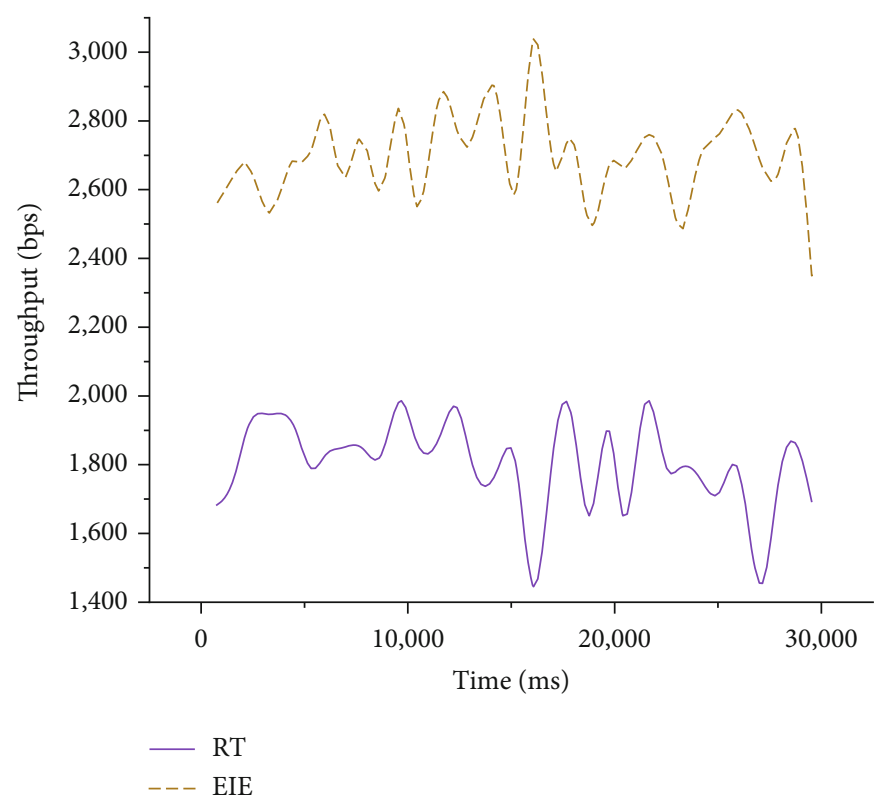

FIGURE 13: Statistics of wireless sensor's communication throughput results in different environments.

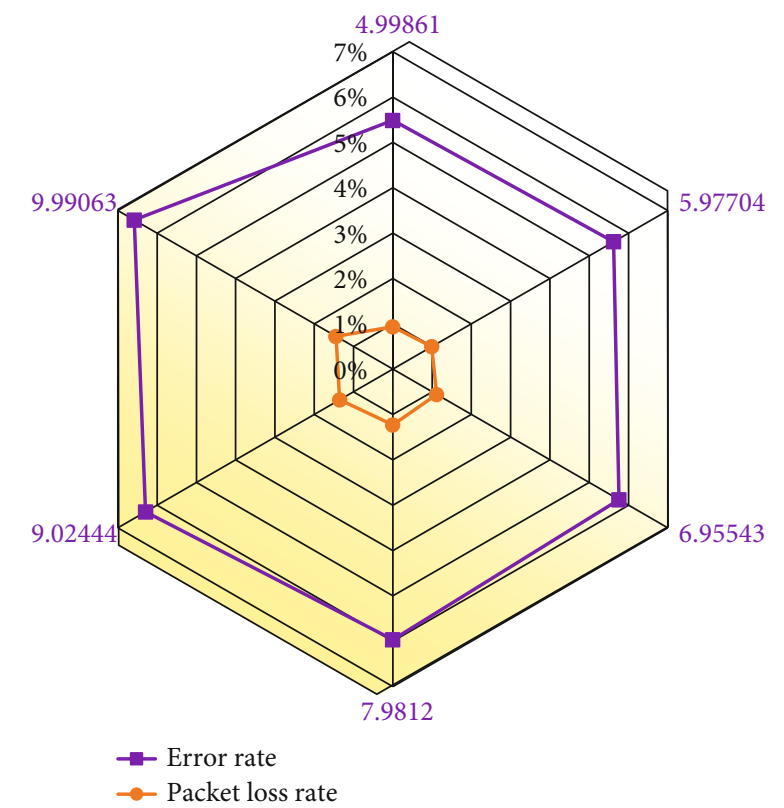

FIGURE 14: Relationship between EMF span and wireless sensor signal's PLR and BER.

between PLR and BER is not apparent when the EMF INT is consistent.

Figure 15 plots the relationship between the PLR and BER when the EMF span is $5 \mathrm{~m}$.

Figure 15 corroborates that when the EMF span of the wireless sensor signal is set to 5 meters, the BER continues to change and shows an upward trend with the increase of the EMF INT at the transmitting end. In contrast, the PLR has been between $0.9 \%$ and $1 \%$, and the data change is not obvious. Therefore, the evolution of field INT at the transmitting end of the wireless sensor signal significantly impacts the BER but has little impact on the PLR. The rea-

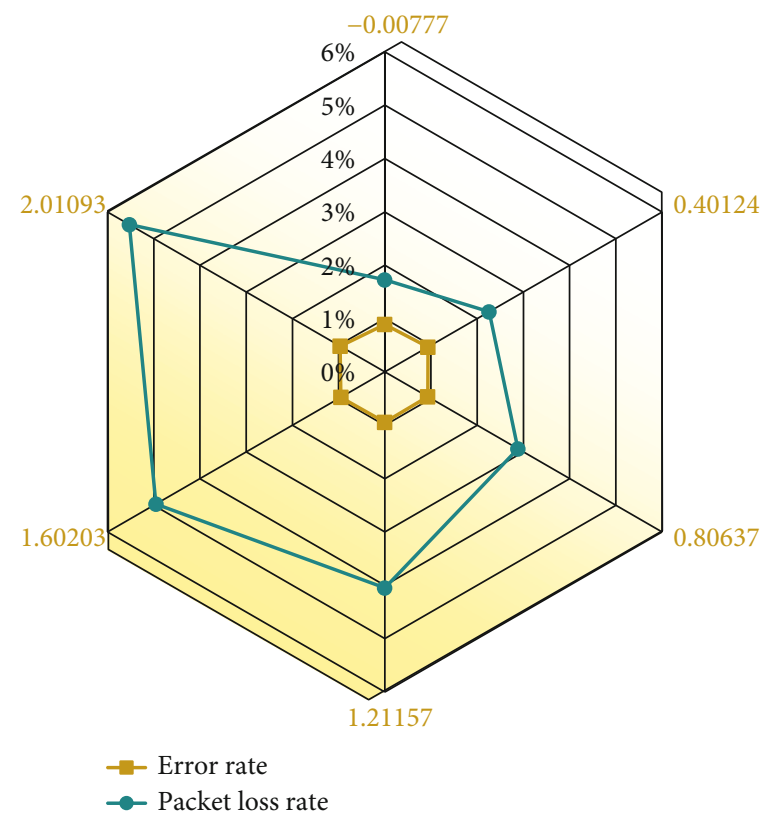

FIgure 15: Changes of BER and PLR when the EMF span is $5 \mathrm{~m}$.

son may be the influence of wireless sensors' internal circuits.

The above experimental analysis concludes that EMF will have varying degrees of impact on wireless sensor SP performance to a certain extent. It is necessary to study the anti-EM performance of wireless sensor SP to reduce interference. At present, the physics community agrees to use a high polymer epoxy resin to wrap the signal $\mathrm{T} \& \mathrm{R}$ of the wireless sensor to reduce EMI. The method is simple and easy to handle. Still, due to the characteristics of the wireless sensor itself, there can be no inclusions on the surface when transmitting and receiving signals, so it is still difficult to 
fundamentally solve the problem of EMI of the wireless sensor. Therefore, it is necessary to study the EMI from multiple angles.

\section{Conclusion}

With the continuous improvement of social S \& T, WST gets ever more close to daily human life, but sometimes, EMI disrupts its normal operations. Accordingly, this paper studies and analyzes the interference of EMF on wireless sensor SP performance using the comparative analysis method and literature method and mainly draws the following conclusions. (i) This paper mainly studies the influence of EMF on wireless sensor information $\mathrm{T} \& \mathrm{R}$ time, $\mathrm{PRR}$, and communication throughput. In the information $\mathrm{T} \& \mathrm{R}$ time, in the same environment, the receiving time is prolonged by about 42,000 ms compared with the wireless sensor not affected by EMI. Under different environments, the interference of EMF will extend the receiving time of wireless sensor information by about $66,000 \mathrm{~ms}$. (ii) In the same environment, the PRR of wireless sensors disturbed by EMF fluctuate between $35 \%$ and $81 \%$. The PRR of wireless sensor packets disturbed by EMF fluctuates between $10 \% \sim 68 \%$ in different environments. (iii) In the same environment, the communication throughput disturbed by EMF is between 1,700 and 2,300. In different environments, the wireless sensor communication throughput affected by EMF is between 1,400 and 2,100 . (iv) When the transmitting and receiving field INTs are consistent, there is a linear relationship between the EMF span and the PLR and BER of the wireless sensor signal. Still, the difference between the final PLR and BER is not apparent. When the distance between the transmitter and receiver of the wireless sensor signal is set to $5 \mathrm{~m}$, the change of field INT at the transmitter significantly impacts the BER but has little impact on the PLR. This paper analyzes the SP performance of the wireless sensor in the EMI environment, which provides specific method guidance for the relevant research in this field.

Still, this paper has some limitations in data acquisition, resulting in deviations in data inspection. Additionally, the research on wireless sensor SP performance in the EMI environment has not been discussed regarding economic investment. In the future, the benefit can be evaluated according to the specific situation to reduce the influence of EMF on the wireless sensor SP performance.

\section{Data Availability}

The data used to support the findings of this study are included within the article.

\section{Conflicts of Interest}

The authors declare that they have no conflicts of interest.

\section{References}

[1] D. Tripathi, J. Prakash, A. K. Tiwari, and R. Ellahi, "Thermal, microrotation, electromagnetic field and nanoparticle shape effects on Cu-CuO/blood flow in microvascular vessels," Microvascular Research, vol. 132, no. 78, p. 104065, 2020.

[2] L. Meng, Y. Wang, M. Gao, and M. Sun, "Electromagnetic field gradient-enhanced Raman scattering in TERS configurations," The Journal of Physical Chemistry C, vol. 125, no. 10, pp. 56845691, 2021.

[3] S. Adda, T. Aureli, S. D'elia et al., "A theoretical and experimental investigation on the measurement of the electromagnetic field level radiated by $5 \mathrm{G}$ base stations," IEEE Access, vol. 8, no. 15, pp. 101448-101463, 2020.

[4] N. Mehran, S. Jafari, S. M. Golpayegani, F. Nazarimehr, and M. Perc, "Different synaptic connections evoke different firing patterns in neurons subject to an electromagnetic field," Nonlinear Dynamics, vol. 100, no. 2, pp. 1809-1824, 2020.

[5] D. Yu, S. Samimi, A. Vasheghani-Farahani, S. P. Shariatpanahi, P. Abdolmaleki, and A. M. Ansari, "Electromagnetic field therapy in cardiovascular diseases; a review of patents, clinically effective devices, and mechanism of therapeutic effects," Trends in Cardiovascular Medicine, vol. 36, no. 11, p. 936, 2020.

[6] X. Abdel, Y. Zhang, Y. Guo et al., "Electromagnetic fieldprogrammed magnetic vortex nanodelivery system for efficacious cancer therapy," Advanced Science, vol. 8, no. 18, p. $2100950,2020$.

[7] L. Li, K. Y. Bliokh, F. Nori, and J. Dressel, “Acoustic versus electromagnetic field theory: scalar, vector, spinor representations and the emergence of acoustic spin," New Journal of Physics, vol. 22, no. 5, article 053050, 2019.

[8] V. Mildaziene, A. Ivankov, G. Pauzaite et al., "Seed treatment with cold plasma and electromagnetic field induces changes in red clover root growth dynamics, flavonoid exudation, and activates nodulation," Plasma Processes and Polymers, vol. 18, no. 1, p. 2000160, 2021.

[9] A. Kacprzyk, G. Kanclerz, E. Rokita, and G. Tatoń, "Which sources of electromagnetic field are of the highest concern for electrosensitive individuals? Questionnaire study with a literature review," Electromagnetic Biology and Medicine, vol. 40, no. 1, pp. 33-40, 2021.

[10] A. Shapourzadeh, S. M. Atyabi, S. Irani, and H. Bakhshi, "Osteoinductivity of polycaprolactone nanofibers grafted functionalized with carboxymethyl chitosan: synergic effect of $\beta$-carotene and electromagnetic field," International Journal of Biological Macromolecules, vol. 150, no. 10, pp. 152-160, 2020.

[11] S. D. Dutta, T. Park, K. Ganguly et al., "Evaluation of the sensing potential of stem cell-secreted proteinsviaa microchip device under electromagnetic field stimulation," ACS Applied Bio Materials, vol. 4, no. 9, pp. 6853-6864, 2021.

[12] A. Franczak, E. M. Waszkiewicz, W. Kozlowska, A. Zmijewska, and A. Koziorowska, "Consequences of electromagnetic field (EMF) radiation during early pregnancy - androgen synthesis and release from the myometrium of pigs _in vitro_," Animal Reproduction Science, vol. 218, no. 29, p. 106465, 2020.

[13] M. Parmaksiz, Ö. Lalegül-Ülker, M. T. Vurat, A. E. Elçin, and Y. M. Elçin, "Magneto-sensitive decellularized bone matrix with or without low frequency- pulsed electromagnetic field exposure for the healing of a critical-size bone defect," Materials Science and Engineering: C, vol. 124, no. 57, p. 112065, 2021.

[14] Y. Z. Zhang, Y. D. Chen, Q. D. Qin, and L. Wei, "Synthesis of FeCoNiCuZn single-phase high-entropy alloy by highfrequency electromagnetic-field assisted ball milling," Journal 
of Magnetism and Magnetic Materials, vol. 498, no. 17, p. 166151, 2020.

[15] D. Franci, S. Coltellacci, E. Grillo et al., "Experimental procedure for fifth generation (5G) electromagnetic field (EMF) measurement and maximum power extrapolation for human exposure assessment," Environments, vol. 7, no. 3, p. 22, 2020.

[16] X. F. Bai, W. Xin, and X. X. Liu, "Asymmetric Gaussian confinement potential and decoherence effect on polaron in quantum disk with electromagnetic field," The European Physical Journal Plus, vol. 135, no. 3, pp. 1-15, 2020.

[17] F. Salek, J. Baharara, K. N. Shahrokhabadi, and E. Amini, "The guardians of germ cells; Sertoli-derived exosomes against electromagnetic field-induced oxidative stress in mouse spermatogonial stem cells," Theriogenology, vol. 173, no. 53, pp. 112122, 2021.

[18] T. Liang and Y. Z. Xie, "Determining incidence and polarization of electromagnetic field for maximal/minimal coupling to transmission line system," IEEE Microwave and Wireless Components Letters, vol. 30, no. 11, pp. 1021-1024, 2020.

[19] W. Sabat, D. Klepacki, K. Kamuda, and K. Kurylo, "Analysis of electromagnetic field distribution generated in an semianechoic chamber in aspect of RF harvesters testing," IEEE Access, vol. 9, no. 11, pp. 92043-92052, 2021.

[20] A. Mariscotti, “Assessment of human exposure (including interference to implantable devices) to low-frequency electromagnetic field in modern microgrids, power systems and electric transports," Energies, vol. 14, no. 20, p. 6789, 2021.

[21] J. H. Kim, D. J. Kang, J. S. Bae et al., "Activation of matrix metalloproteinases and FoxO3a in HaCaT keratinocytes by radiofrequency electromagnetic field exposure," Scientific Reports, vol. 11, no. 1, pp. 1-10, 2021.

[22] F. Pacchierotti, L. Ardoino, B. Benassi et al., "Effects of radiofrequency electromagnetic field (RF-EMF) exposure on male fertility and pregnancy and birth outcomes: protocols for a systematic review of experimental studies in non-human mammals and in human sperm exposed_in vitro_," Environment International, vol. 157, no. 19, p. 106806, 2021.

[23] V. V. Bezpal'ko, S. V. Stankevych, L. V. Zhukova et al., "Presowing treatment of winter wheat and spring barley seeds with the extremely high frequencies electromagnetic field," Ukrainian Journal of Ecology, vol. 11, no. 1, pp. 62-71, 2021.

[24] A. Trzyna, B. Pikuła, A. Ludwin, B. Kocan, and A. Banaś-Ząbczyk, "The influence of an electromagnetic field on adiposederived stem/stromal cells' growth factor secretion: modulation of FGF-2 production by in vitro exposure," Archives of Biological Sciences, vol. 72, no. 3, pp. 339-347, 2020.

[25] E. Marshalko, M. Kruglyakov, A. Kuvshinov et al., "Comparing three approaches to the inducing source setting for the ground electromagnetic field modeling due to space weather events," Space Weather, vol. 19, no. 2, p. 657, 2021.

[26] N. Agrawal, K. Verma, D. Baghel et al., "Effects of extremely low-frequency electromagnetic field on different developmental stages ofDrosophila melanogaster," International Journal of Radiation Biology, vol. 97, no. 11, pp. 1606-1616, 2021.

[27] G. M. Andolina, F. M. D. Pellegrino, V. Giovannetti, A. H. MacDonald, and M. Polini, "Theory of photon condensation in a spatially varying electromagnetic field," Physical Review $B$, vol. 102, no. 12, p. 125137, 2020.

[28] P. Song, Z. Cui, Y. Hui, W. Zhao, J. Wang, and Y. Han, "Explicit analytical expressions for the electromagnetic field components of typical structured light beams," Journal of
Quantitative Spectroscopy and Radiative Transfer, vol. 241, no. 38, p. 106715, 2020.

[29] H. S. Kim, H. D. Choi, J. K. Pack, N. Kim, and Y. H. Ahn, "Biological effects of exposure to a radiofrequency electromagnetic field on the placental barrier in pregnant rats," Bioelectromagnetics, vol. 42, no. 3, pp. 191-199, 2021.

[30] A. Cios, M. Ciepielak, W. Stankiewicz, and Ł. Szymański, "The influence of the extremely low frequency electromagnetic field on clear cell renal carcinoma," International Journal of Molecular Sciences, vol. 22, no. 3, p. 1342, 2021.

[31] E. M. Drzewiecka, W. Kozlowska, A. Zmijewska, P. J. Wydorski, and A. Franczak, "Electromagnetic field (EMF) radiation alters estrogen release from the pig myometrium during the peri-implantation period," International Journal of Molecular Sciences, vol. 22, no. 6, p. 2920, 2021.

[32] D. Duncan and I. Dinev, "Noninvasive induction of muscle fiber hypertrophy and hyperplasia: effects of high-intensity focused electromagnetic field evaluated in an in-vivo porcine model: a pilot study," Aesthetic Surgery Journal, vol. 40, no. 5, pp. 568-574, 2020.

[33] P. N. D'yachkov and E. P. D'yachkov, "Propagation of an electromagnetic field along the axis of metal carbon nanotubes," Russian Journal of Inorganic Chemistry, vol. 66, no. 3, pp. 367-373, 2021. 